\section{Real-Life-Daten bestätigen Überlebensvorteil von SCLC-Patienten unter Chemotherapie}

\author{
Für Patienten mit kleinzelligem Lungenkarzinom (SCLC) stehen mehrere \\ Chemotherapie-Regimes zur Verfügung. Doch verändern die positiven \\ Resultate aus Studien auch die Behandlungsrealität? In einer Register- \\ analyse wurde dieser Frage nachgegangen.
}

der Zeitraum von 1985-1990. Mit ihm wurden die folgenden Jahre in 5-JahresIntervallen bis 2005 (1991-1995, 19962000, 2001-2005) bezüglich des Gesamtüberlebens verglichen.

Die CT-Raten stiegen mit Werten von $38,55,50$ und $53 \%$ signifikant an. Alter und Tumorstadium sowie Wohnort und ethnische Herkunft des Patienten waren mit der Wahl einer CT signifikant assoziiert. Frauen, Asiaten und in ländlichen Gebieten Lebende erhielten häufiger eine CT. Das mediane Gesamtüberleben betrug mit CT 9,6, ohne CT 3,6 Monate, in linearen Trendanalysen über den gesamten Zeitraum war der Überlebensvorteil durch die zusätzliche CT nachweisbar. Innerhalb der Gruppe chemotherapeutisch behandelter Patienten ließen sich im Ergebnis keine Unterschiede zwischen einer Carboplatin- und einer Cisplatin-

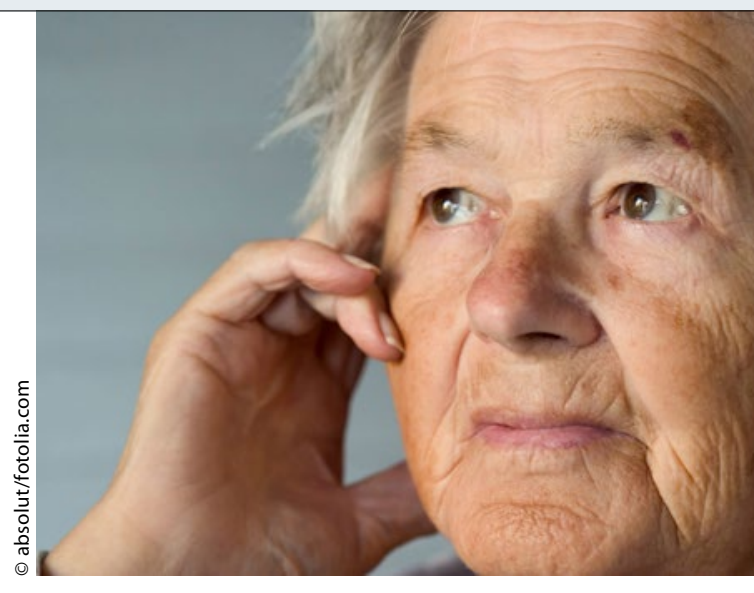

Frauen erhielten etwas häufiger eine Chemotherapie als Männer.

Therapie ausmachen. Eine zusätzlich zu den Platintherapien durchgeführte CT verbesserte das Überleben nochmals.

Fazit: SCLC-Patienten, die im klinischen Alltag außerhalb von Studien behandelt werden, profitieren ebenso wie Studienpatienten von einer Chemotherapie. Neuere Substanzen verbesserten die Prognose zusätzlich. Insgesamt wird die Chemotherapie beim SCLC aber immer noch zurückhaltend eingesetzt.

Barbara Kreutzkamp

Behera $\mathrm{M}$ et al. Trends, predictors, and impact of systemic chemotherapy in small cell lung cancer patients between 1985 and 2005. Cancer. 2016;122(1):50-60.

\section{Frühes SCLC: Vorteile für adjuvante Chemotherapie mit oder ohne kraniale RT?}

\begin{abstract}
Patienten mit kurativ resezierbarem kleinzelligem Lungenkarzinom (SCLC) haben nach zusätzlicher Radio(chemo-)therapie eine bessere Überlebensprognose. Eine bevölkerungsbasierte Registerstudie liefert konkrete Zahlen.
\end{abstract}

\footnotetext{
Datienten mit frühem, resektablem SCLC sollten postoperativ eine adjuvante Chemotherapie (CT) erhalten. Allerdings steht diese Empfehlung der NCCN(National Comprehensive Cancer Network)-Leitlinie noch auf wackeliger Studienbasis. Auch zum Nutzen einer prophylaktischen kranialen Bestrahlung (PCI) fehlen Studien. Neue Zahlen generierten jetzt US-amerikanische Kollegen mithilfe der National Cancer Data Base anhand der Daten von 954 Patienten mit einem komplett resezierten SCLC im Stadium T1-2 N0M0. Sie waren in den
}

Jahren 2003-2011 nach der Operation nicht adjuvant oder mit adjuvanter CT und/oder Radiotherapie (RT) behandelt worden, hatten mindestens 30 Tage überlebt und waren median über $43 \mathrm{Mo-}$ nate nachbeobachtet worden.

Die Rate für das 5-Jahres-Überleben lag bei $47 \%$. Nach der Resektion hatten $59 \%$ der Patienten eine adjuvante Behandlung erhalten - 37,1\% eine alleinige CT, 9,9\% eine Radiochemotherapie (davon etwa die Hälfte zusätzlich eine $\mathrm{PCI}$ ) sowie 2,3\% eine alleinige RT, entweder kranial $(n=5)$ oder thorakal
( $\mathrm{n}=17$ ). Eine adjuvante CT mit oder ohne begleitende RT verbesserte das Gesamtüberleben im Vergleich zur alleinigen Operation signifikant (median 66,0 vs. 42,1 Monate; $\mathrm{p}<0,01$ ). Die multivariate Analyse bestätigte das: Die Hazard Ratio für das Gesamtüberleben bei zusätzlicher CT betrug 0,78 (95\%-Konfidenzintervall [95\%-KI] 0,63-0,95) und bei adjuvanter CT plus zusätzlicher PCI sogar 0,52 (95\%-KI 0,36-0,75) - jeweils im Vergleich zu alleiniger Resektion.

Fazit: Patienten mit komplett reseziertem SCLC im Stadium T1-2 N0 M0 haben durch eine adjuvante Chemotherapie mit und ohne kraniale Bestrahlung eine bessere Überlebensprognose.

\section{Barbara Kreutzkamp}

Yang CFJ et al. Role of adjuvant therapy in a population-based cohort of patients with early-stage small-cell lung cancer. J Clin Oncol. 2016;34(10):1057-64. 